\title{
Cystodistension injuries and long-term bladder functional outcomes: A unique case series from medical malpractice
}

\begin{abstract}
$\underline{\text { Abstract }}$
Objectives: Bladder over-distension injuries are rare, but can have devastating effects on the individual and lead to litigation. We aimed to (a) test the hypothesis: larger distension volumes and longer distension times are more likely to generate acontractile bladders (b) determine prognostic information (c) guide management protocols aimed at preventing cystodistension injuries.
\end{abstract}

Patients and Methods: We report 18 cases of cystodistension injury following acute urinary retention. One author provided expert medical opinion in these cases, who had sought redress in the UK Courts through medical malpractice claims. Data was collected retrospectively.

Results: Cohort comprised 17 females, 1 male. Mean age 31 years (range 17-69 years). Precipitating events included 6 surgical procedures, 11 vaginal deliveries and 1 episode of back pain. Median retention volume was $1450 \mathrm{ml}$ (range 1000-3800 ml), median duration of retention was 1200 minutes (range 470-10365 minutes). Fourteen patients performed interment self catheterisation, 6 requiring this temporarily. No statistically significant association was demonstrated between retention characteristics and long-term bladder functional outcomes.

Conclusions: This case series demonstrated cystodistension injuries to be unpredictable in their long-term impact. No association between volume and duration of retention and long-term bladder function was found. Long-term $50 \%$ void spontaneously, $33 \%$ void spontaneously but require ISC to effect complete bladder emptying and $17 \%$ remain catheter dependant.

Keywords: Bladder overdistension, underactive, urinary retention

Level of evidence: Level 4 


\section{Introduction}

It is believed that bladder distension leads to temporary damage to nerve terminals within the detrusor muscle of the bladder via the effect that bladder distension has on the detrusor blood supply. Overdistension of the bladder causes a rise in bladder pressure which in turn reduces detrusor blood supply. If bladder overdistension is maintained over several hours then detrusor blood flow can be sufficiently reduced such that ischaemic damage to the sensory and motor nerve supply of the bladder can occur. Ischaemia leads to the release of reactive oxygen species which mediate nerve injury. ${ }^{1,2}$

The clinical consequence of this nerve injury is diminished capacity to generate a sustained bladder contraction and therefore sustained bladder pressure during attempted bladder emptying, such that bladder emptying is incomplete, and the patient leaves a residual urine volume after each void. It also leads to loss of the ability to sense bladder filling and bladder fullness, consequent on damage to the bladder sensory nerves. Even a single episode of overdistension can permanently damage the detrusor muscle and result in the permanent requirement for daily intermittent self catheterisation (ISC). ${ }^{3}$

With normal functional bladder capacity ranging from approximately 400 to $600 \mathrm{ml}$, volumes greater than $600 \mathrm{ml}$ can generate pain or discomfort in a neurologically intact individual. ${ }^{4}$ This pain or discomfort arising from the neuro-sensory feedback loop of the bladder protects against overdistension injuries.

Bladder over distension is an important albeit uncommon medical complication seen most frequently, in our experience, in the context of obstetric practice especially where regional anaesthesia has been used during labour or after extensive pelvic or orthopaedic surgery. ${ }^{5}$ Anaesthetic agents temporarily reduce detrusor motor and bladder sensory function, allowing large retention volumes to go undetected.

Voiding problems following childbirth are well recognised. Postpartum voiding dysfunction, defined as failure to pass urine spontaneously within 6 hours of vaginal delivery or catheter removal after delivery, is said to occur in $0.7-4 \%$ of deliveries. ${ }^{6} \mathrm{~A}$ single episode of over distension due to delayed diagnosis can cause bladder injury and lead to persistent voiding difficulties. If voiding difficulties are recognised early and adequately managed, both short- and long-term morbidity can be prevented. The post-partum bladder has a tendency to be underactive and is therefore vulnerable to retention of urine (especially where regional anaesthesia has been used during labour) following trauma to the bladder, pelvic floor muscles and nerves during delivery. ${ }^{7}$ In the post-partum patient, difficulties 
voiding can be exacerbated by hormonal changes in pregnancy causing reduced detrusor tone, and so bladder capacity increases from the third month of pregnancy. This increase may not be obvious to the pregnant woman due to pressure from the enlarging uterus, but after delivery the reduced detrusor tone can cause short-term difficulties in voiding. ${ }^{8}$

The UK's National Institute for Clinical Excellence (NICE) Intrapartum Care guidelines mandate that no labouring woman should pass 6 hours without voiding urine. ${ }^{9}$ Upon the 6 hour limit being reached, either a bladder scan or in-out catheterisation must be carried out to quantify the volume of urine present and thereby protect the bladder against a distension injury.

Given the paucity of evidence on the management of postpartum urinary retention, many hospitals have implemented their own postpartum bladder care protocols, but this has led to variations in diagnosis and management. ${ }^{7}$

In an attempt to increase awareness of this condition, detail its impact on individual patients and guide future management protocols aimed at preventing cystodistension injuries, we report on 18 cases of cystodistension injuries following acute urinary retention.

\section{Patients and Methods}

Between January 2002 and April 2016, one of the authors (JR) was asked to provide an opinion on causation, condition and prognosis in 18 cases of cystodistension injury, where the patients affected had sought redress in the UK Courts through a medical malpractice claim. Each Claimant successfully won their case. All cystodistension events occurred in UK hospitals. Retrospective data collection and analysis of the 18 cases was conducted from review of the medico-legal documentation (including the medical records) involved in the legal proceedings.

For the purposes of this study the retention volume was defined as the initial volume drained upon urethral catheterisation to relieve the retention.

\section{$\underline{\text { Results }}$}

The cohort comprised 17 female and 1 male patient. The mean age was 31 years (range 17-69 years). The precipitating clinical events included 6 surgical procedures, 11 vaginal deliveries and 1 
episode of back pain requiring admission for opiate analgesia. The 6 surgical procedures were 2 lower limb orthopaedic operations ( 1 total hip replacement and 1 foot osteotomy) and 4 intraabdominal gynaecological operations (1 laparoscopic salpingo-ophorectomy, 1 subtotal abdominal hysterectomy for fibroids and 2 diagnostic laparoscopies with one requiring adhesiolysis and ovarian cyst drainage). All 6 operations were carried out under general anaesthesia, combined in 1 orthopaedic case with a femoral nerve block. The 11 vaginal deliveries involved 5 instrumental deliveries with forceps and 6 spontaneous vaginal deliveries (SVD). No patients underwent caesarean section. Nine of the 11 vaginal deliveries received epidurals including all of the 5 instrumental deliveries and 4 of the SVDs.

Thirteen patients had no previous urological history. Two had experienced recurrent urinary tract infections prior to the injury. The 1 male patient reported nocturia as a longstanding, stable symptom. Two had a history of retention and altered bladder sensation following prior vaginal deliveries, but were subsequently asymptomatic.

The median retention volume was $1450 \mathrm{ml}$ (interquartile range 1200-2000, range 1000-3800 ml). Duration of retention was classified as the time period from last normal void (>150 ml), or removal of the initial catheter, to insertion of a urethral catheter. The median duration of retention was 1200 minutes i.e. 20 hours (interquartile range 650-2070, range 470-10365 minutes).

During the retention period, 14 patients voided volumes of less than $100 \mathrm{ml}$. The frequency of these small voided volumes $(<100 \mathrm{mls})$ ranged between $1-6$. These 14 individuals therefore appeared, on the face of it, to be voiding spontaneously, but were actually in a marked state of urinary retention.

When examined by JR all 18 patients had normal sacral sensation and normal bulbocavernosus and anocutaneous reflexes indicating normal S3-5 sensation, an intact sacral cord and intact sacral motor nerve roots. ${ }^{10}$

\section{Immediate management}

All patients were initially managed with a period of urethral catheterisation. First attempt at trial without catheter (TWOC) occurred, at a mean, on day 6 after insertion (range 1-18). Fifteen patients (83\%) failed the initial TWOC. The 3 patients that passed their initial TWOC had a median retention volume of $1500 \mathrm{mls}$ (range $1000-2000 \mathrm{mls}$ ). The 15 patients that failed their initial TWOC had a mean retention volume of $1400 \mathrm{ml}$ (range $1000-3800 \mathrm{mls}$ ). There was no statistically significant difference in retention volumes between the groups ( $p=0.86$, using Kruskal-Wallis test). Duration of retention for the 3 patients that passed their TWOC was a median of 2407 minutes (range 500-2580 mins), and 
960 minutes (range 470-10365 minutes) for the 15 that failed their TWOC ( $p=0.59$, i.e. no statistically significant difference by failure of initial TWOC).

\section{Initial management}

After failing TWOC's, 12 (80\%) patients were taught to perform ISC. One patient remained with an indwelling catheter for 4 months, until learning ISC. Two patients underwent supra-pubic catheterisation (SPC). One patient with an SPC went on to learn ISC and SPC removal, 21 months after the SPC was inserted.

\section{Did distension volume and duration impact on functional bladder outcomes?}

When evaluating the average distension volumes and durations by long-term outcome, somewhat surprisingly patients spontaneously voiding had the longest and largest distension characteristics.

The median (range) retention volume was 1500 (100-3400) $\mathrm{ml}$ in patients spontaneously voiding, 1200 (1100-1500) $\mathrm{ml}$ in those who were fully catheter dependent and 1275 (1000-3800) $\mathrm{ml}$ in those using a mixture of ISC and spontaneous voiding. There was no evidence of a statistically significant difference between the outcome groups by initial retention volume $(p=0.37$, using a 3-sample equality of medians test). The relationship between distension volume $<2000 \mathrm{vs}$. $\geq 2000 \mathrm{ml}$ and functional bladder outcome (by need for catheterisation) is shown in Table 1. When the volume of retention was $<2000 \mathrm{ml}$, spontaneous voiding was achieved in $42 \%$, a combination of spontaneous voiding and ISC was required to effect bladder emptying in $33 \%$ and $25 \%$ remained completely ISC or indwelling catheter dependent. With a retention volume of $\geq 2000 \mathrm{mls}$, spontaneous voiding was achieved in 67\%, a combination of spontaneous voiding and ISC was required to effect bladder emptying in $33 \%$ and $0 \%$ remained catheter dependent.

The median (range) duration of retention was 1749 (500-10365) minutes in patients spontaneously voiding, 1440 (540-2070) minutes in those who were fully catheter dependent and 743 (470-1440) minutes in those using a mixture of ISC and spontaneous voiding. There was no evidence of a statistically significant difference between the outcome groups by duration of retention $(p=0.14)$. The relationship between duration of distension and functional bladder outcome is shown in Table 2. When the duration of retention was $<1000$ minutes, spontaneous voiding was achieved in $33 \%$, a 
combination of spontaneous voiding and ISC was required to effect bladder emptying in $56 \%$ and $11 \%$ remained completely ISC or indwelling catheter dependent. When the duration of retention was $\geq 1000$ minutes, spontaneous voiding was achieved in $67 \%$, both ISC and spontaneous voiding was required in $11 \%$ and $22 \%$ remained catheter dependent.

\section{Urodynamic outcomes}

Urodynamic studies were performed in $9(50 \%)$ of the patients. Urodynamic findings demonstrated acontractile bladders in 3 patients. Impaired detrusor function that was inadequate to generate a pressure to void normally was demonstrated in 3 patients. Adequate detrusor ability to void appropriately was demonstrated in 3 patients.

Individuals with an acontractile bladder had median (range) retention volumes of 1400 (1100-2000) $\mathrm{ml}$ and durations of retention of $960(885-1440)$ minutes. The 3 patients whose bladders were capable of generating sufficient detrusor pressures to effect complete emptying had a median (range) retention volume of 1300 (1000-1300) $\mathrm{ml}$ and duration of retention 836 minutes (range 4701749 minutes). There was no evidence for a difference between the two groups by volume or duration of retention (Kruskall-Wallis $p=0.27$ and $p=0.51$ respectively). Patients with impaired detrusor function (but not acontractile) bladders (demonstrating a degree detrusor contraction, but insufficient to void to completion) had median (range) retention volumes of $1200 \mathrm{ml}$ (range 1000$1500 \mathrm{ml})$ and durations of retention of 650 (540-2070) minutes.

\section{Sacral Nerve Modulators}

Three patients received sacral nerve modulators (SNM - Medtronic Interstim) placed on average 21 months after the distension injury was sustained (range 14-28 months). At final follow up 2 were voiding spontaneously but also performing ISC to effect complete bladder emptying (with residual volumes averaging $200 \mathrm{ml}$ in both cases). One patient was voiding spontaneously, with residual volumes $<100 \mathrm{ml}$.

\section{Long term outcomes}

Final follow up averaged 29 months from injury (range 5-45 months). At this point in time nine patients (50\%) were spontaneously voiding (one of whom had undergone SNS implantation). Six 
patients (33\%) were spontaneously voiding and performing ISC to effect complete bladder emptying (two of whom had undergone SNS implantation). One patient (6\%) remained with a long term SPC. Two patients (11\%) remained completely ISC dependent.

Long term requirements for ISC varied. Of the 14 patients that performed ISC as a result of a bladder distension injury, 6 (43\%) required ISC temporarily (this temporary requirement for ISC lasted a mean of 383 days from injury - range $18-1080$ days). Six patients (43\%) continued to perform ISC in addition to spontaneous voiding. Two patients (14\%) remained ISC dependent at 28 and 24 months after injury.

\section{Discussion}

We believe this is the first series to document short- and long-term functional bladder outcomes in individuals who have sustained a bladder distension injury. When examined all 18 patients had evidence of an intact sacral cord and intact sacral motor nerve roots. ${ }^{10}$ There was therefore no evidence of sacral neuropathy to account for their subsequent voiding dysfunction. The cause of their subsequent loss of bladder sensory function and difficulty voiding urine was therefore likely to be a bladder distension injury.

We identified that the minimum retention volume capable of causing a distension injury was $1000 \mathrm{ml}$ (in this series of 18 individuals pursuing a clinical negligence claim) and the minimum duration of retention required to cause such an injury was 470 minutes (just 8 hours). It is conceivable that lower retention volume may be capable of causing a distension injury, but perhaps of lesser severity such that individuals decide not to litigate.

We hypothesised that individuals with larger distension volumes and longer distension times would be more likely to end up with an acontractile bladder and therefore permanently ISC-dependant. Such a hypothesis seems intuitively reasonable, however we did not find evidence to support this. Within the limits of the power of the study, we were unable to demonstrate any statistically significant association between distension characteristics (volume and duration) and long-term bladder function (the maintenance of spontaneous voiding function or the requirement for catheterisation - intermittent or permanent) (Table 1 and 2). Similarly, we did not find an association between failure of initial TWOC and distension characteristics either.

The lack of association between severity of bladder distention and long-term outcomes, suggests additional factors affect the extent of the tissue damage, response, and subsequent altered bladder 
function experienced by individuals who have suffered a distension. There may be individual variations in the susceptibility or resilience to a distension injury to account for some individuals with high distension volumes and prolonged distension times having a good functional outcome.

This hypothesis is supported by the finding that 2 patients who remained ISC dependent had low distension volumes of just $1100 \mathrm{ml}$ and $1200 \mathrm{ml}$ and relatively short distension durations of 1440 minutes and 540 minutes respectively. This compares with an median distension volume of $1450 \mathrm{ml}$ and median duration of distension of 1200 minutes.

Conversely, when comparing the average distension volumes and durations with long term outcomes, those individuals who ultimately were able to void urine spontaneously, without the need to do ISC, had a higher average distension volume and longer distension duration than those who were ISC dependant or were able to void spontaneously but required ISC for complete bladder emptying.

While there may be a threshold at which such injuries can occur (apparently $1000 \mathrm{ml}$ in our series, but it is conceivable that lower retention volumes may be capable of causing a distension injury), individual bladder resilience means that long-term outcome is unpredictable. From our series we can reassure patients that irrespective of duration or volume of distension, $50 \%$ will recover enough function not to require any form of catheterisation and only $17 \%$ will remain completely catheter (ISC or indwelling) dependant.

Temporary ISC was required in $43 \%$ of individuals, before returning to spontaneous voiding. Temporary requirements for ISC lasted a mean of 383 days (range 18-1080). Again this provides useful prognostic information.

The ability to void small volumes during the period of overdistension in $82 \%$ of the cohort demonstrates the need for post-partum bladder care guidelines to include and specify on the need to perform post-void residual volume measurements, to eliminate the potential danger of being falsely reassured that a patient is not in retention, based on small and frequent voided volumes that could mask a large persistence post-void residual volume.

Perhaps surprisingly, given that a distension injury damages the intramural innervation of the bladder, one of three patients who underwent implantation of a Medtronic Interstim sacral nerve modulator was able to void spontaneously without recourse to ISC. The other two patients who underwent implantation of a Medtronic Interstim sacral nerve modulator were able to void some urine spontaneously, but remained ISC dependant for complete bladder emptying. For these two 
patients, prior to insertion of the sacral nerve modulator, one patient already spontaneously voided but required ISC to drain a residual volume of $200 \mathrm{ml}$. The second patient was entirely ISC dependent._The implants in these individuals took place on average 21 months after the distension injury range 14-28 months, suggesting that the implant achieved improved bladder function, since we would have expected any spontaneous recovery of bladder function to have occurred within 14 months and certainly by 28 months post-distension injury. At final follow up the one patient voided spontaneously and had residual volumes of $<100 \mathrm{ml}$ and the two patients still performing ISC had average residual volume of $200 \mathrm{ml}$. Had the remaining 7 patients who failed to regain the ability to void spontaneously been offered SNS, they may have benefited from this approach.

Recent work from Levanovich PE et al. has demonstrated encouraging early results in non neurogenic patients suffering with underactive bladder. ${ }^{11}$ Their work describes improved bladder function after intradetrusor injection of autologous muscle-derived cells, harvested from needle biopsy of quadriceps femoris. This early work has the potential to provide greater understanding of the physiological response of the bladder to overdistension, and provide a therapeutic method to treat the sustained bladder injury and functional impairment.

\section{Conclusions:}

This case series demonstrated cystodistension injuries to be unpredictable in their long-term impact on bladder function. Volume and duration of retention do not correlate with long term bladder function.

Overall individuals can be reassured that $50 \%$ will be able to void urine spontaneously without the need for ISC, a further $33 \%$ will be able to void urine spontaneously but will require ISC a number of times daily to effect complete bladder emptying and only $17 \%$ will be completely ISC or suprapubic catheter dependant.

Nonetheless, such injuries have serious implications for both patient and doctor. From the patient's perspective bladder distension injuries have the potential to cause significant functional harm, rendering some permanently catheter dependent. The clinician may potentially be exposed to an allegation of medical malpractice. While our experience provides useful prognostic information for 
such patients, the outcomes demonstrated in this series also highlight the need for post-partum and post operative bladder care guidelines to incorporate detailed use of post-void residual urine volumes in the post-partum or post-operative period.

\section{REFERENCES:}

1) Ramsden PD, Smith JC, Dunn M, et al. Distension therapy for the unstable bladder: later results including an assessment of repeat distensions. Br J Urol. 48. 623.1976.

2)Brading AF, Greenland JE, Mills IW, et al. Blood supply to the bladder during filling. Scand J Urol Nephrol Suppl 201. 25. 1999.

3) Mayo ME, Lloyd-Davies RW, Shuttleworth KED, et al. The damaged human detrusor: functional and electron microscopic changes in disease. Br J Urol 45. 116. 1973

4) Wyndaele JJ, De Wachter S. Cystometrical sensory data from a normal population: Comparison of two groups of young healthy volunteers examined with 5 years interval. Eur Urol. 42. 34. 2002

5) Madersbacher H, Cardozo L, Chapple C et al. What Are the Causes and Consequences of Bladder Overdistension?: ICI-RS 2011. Neurourol Urodyn. 31.317.2012

6) Kearney R, Cutner A. Postpartum voiding dysfunction.TOG.10.71:2011

7)Oxford University Hospitals NHS Foundation Trust. Postpartum Bladder Management Guidelines. 2013.Available from: http://ouh.oxnet.nhs.uk/Maternity/Lists/Maternity\%20Guidelines/GuidelineDispForm.aspx?List=a599f0e6\%2Da2ea \%2D4377\%2D9dac\%2Dd0f96fde4e0c\&ID=71\&RootFolder=\%2FMaternity\%2FLists\%2FMaternity\%20Guidelines [Accessed 6 August 2019]

8) MacKenzie R. Urinary retention after childbirth. International Continence Society. PDF document available from: https://www.ics.org/Documents/DocumentsDownload.aspx?DocumentID=1679

[Accessed 6 August 2019]

9) National Institute for Health and Care Excellence.Intrapartum care for healthy women and babies, Clinical Guideline CG190. 2014. Available from: https://www.nice.org.uk/guidance/cg190. [Accessed 6 August 2109]

10) Pavlakis AJ, Siroky MB, Goldstein I, et al. Neurourologic findings in conus medullaris and cauda equina injury. Arch Neurol 40. 570. 1983.

11) Levanovich PE, Diokno A, Hasenau DL et al. Intradetrusor injection of adult muscle derived cells for the treatment of underactive bladder: pilot study. Int Urol Nephrol.47.465.2015. 
